Lepr Rev (1992) 63, Supplement, 48s-52s

\title{
Towards the use of decision sciences in leprosy control
}

\author{
J D F HABBEMA, E JOZEFZOON \& \\ G J VAN OORTMARSSEN
}

\section{Introduction}

The planning and evaluation of disease control is extremely complicated because of the many factors and uncertainties that are involved in predicting and explaining trends. In leprosy, for example, the impact of control measures on the epidemiological trends is not precisely known. In spite of this, multidrug therapy (MDT) is now the first-choice treatment for leprosy. In countries where MDT is implemented on a large scale, a marked reduction in the prevalence rate has been achieved. It is also noticeable that treatment compliance is improved. These positive effects are mainly attributed to the short duration and low toxicity of MDT. ${ }^{1}$ But even when MDT is chosen, there are many ways in which MDT-strategies can be organized. Planning and evaluation tools would be most welcome.

At our Institute we have developed methods and tools for assisting decision making in disease control programmes which are based on epidemiological simulation modelling. Based on our experience in the control of non-communicable diseases, we started analysing tropical disease control problems in a joint project with the Onchocerciasis Control Programme (OCP). In this project simulation models have been developed which are now used routinely by the OCP in the planning and evaluation of river blindness control measures.

In this paper we will first summarize our approach to developing and using decision aids, and then consider the possibility of using this approach in leprosy control.

\section{Guidelines and approach}

We suggest, with our experience of over 10 years in epidemiologic modelling, that the following guidelines should be followed in developing model-based decision aids in disease control programmes:

- health outcome should play the central role;

- the models should be based on a rational and medically sound basis;

- resource use, economic and cost-effective calculations should be studied when indicated, but always in relation to the health outcome;

- the primary goal is to support decision making, but a secondary-intimately linked-goal is to increase our knowledge and understanding of the disease and the possibilities for its control; 
- a modelling approach is necessary because many interacting factors play a role in the problems that are to be addressed. High quality data and expert knowledge is necessary in developing a satisfactory model;

- development and application of decision models should be a collaborative enterprise with existing control programmes, involving scientists and health workers from different disciplines.

Control questions that should be addressed by epidemiometric models developed according to the above guidelines include:

- what will be the impact of the current or a proposed control strategy on trends in incidence, prevalence, morbidity and mortality of the disease concerned;

- what are the implications for resource use of executing alternative control strategies;

- can the optimal control strategy for a given amount of resources be indicated;

- what will be the favourable and the adverse effects of a specified control strategy from a public health point of view;

- can our knowledge of the natural history and the epidemiology of the disease be improved, either by careful analysis of available data or by conducting special studies;

- are observed trends during control significantly different from the predictions. And if so, in what way has the control programme to be adapted, if at all?

All the above questions should be answered for the specific situation of the country or area where the control programme is carried out.

A number of conceptual and practical requirements should be satisfied in order to develop models that are able to address such diverse questions. Conceptually, they should have the seemingly contradictory properties of being comprehensive and detailed. They should be appropriate for assessing the effects of different control measures on epidemiological and population health trends. They should also be suitable for detailed data analysis and prediction within a control programme. From an organizational point of view, modelling should be an integral part of the disease control programme. This calls for an approach which is easy to use and which can be adapted to new results and to changes in control policy.

The way in which we have tackled model building has been proven successful for both cancer screening, ${ }^{2,3}$ and onchocerciasis control. ${ }^{4-7}$

\section{Epidemiological models for leprosy control}

The first step in the development of decision-oriented epidemiological models is the description of all factors and their inter-relations that are important for the control of leprosy. These can be classified as follows:

- epidemiological indicators: incidence and prevalence, both of infections and of disability; other indicators are the age-distribution of the incidence, and the distribution of type of leprosy. Leprosy mortality is very low and directly linked to disability and can usually be deleted;

- natural history of leprosy, e.g. self-healing;

- diagnostic tools: intensive use is made of clinical diagnosis, and of bacteriological, histopathological and immunological diagnosis;

- the type of control: multidrug therapy has a high cure rate and is quite promising. Health education and contact tracing helps to decrease the secondary cases. In addition, BCG vaccination provides a certain degree of protection; 
- surveillance (early detection of cases, case surveillance);

- behaviour of patients, e.g. compliance, alertness of symptoms. At the level of the control programme this is expressed as coverage and completion rate;

- effectiveness of therapies. Cure and relapse rates, duration of treatment, impact on transmission.

The way and the detail in which these factors and their inter-relations are taken into account, and the way in which they are embedded in a population-disease-intervention model, determines the possibility of their use for addressing control problems. Lechat ${ }^{8}$ has contributed most to leprosy control modelling. We have considerable experience with so-called microsimulation modelling. ${ }^{2,3,6,7}$ This implies simulation of individual human life histories. Together, the simulated individuals constitute a community, e.g. a village population. In the onchocerciasis control model ONCHOSIM, the life-histories of parasites in man are also simulated individually. Stochastic microsimulation enables a realistic formulation of the mechanisms of transmission, which is important in predicting the epidemiological trends associated with different policy options. When a validated, decision-oriented model has been developed, the important questions concerning leprosy control can be addressed.

\section{LEPROSY CONTROL: QUESTIONS AT STAKE}

\section{Prediction of trends}

Decision analytic tools can be used to predict leprosy-related public health trends concerning the prevalence of registered cases, the incidence of new leprosy cases, cases under treatment, RFT cases, relapse rates, and incidence and prevalence of disability. The predictions can be made both for an existing situation and for different (alternative) control options.

\section{Planning of resource requirements}

The short- and mid-term predictions of incidence and prevalence may in turn be used to make projections of the necessary resources, especially financial, that are needed for leprosy surveillance and control.

\section{Choice of control strategies}

The control methods which give the best public health results when resources are limited can be identified. For example, a detailed investigation of MDT could lead to recommendations to ensure a more efficient use of available resources, by analysing the trade-off between (shorter) duration of MDT-treatment and (more) relapse treatment. On the other hand, the conditions and circumstances that make it possible to attain certain goals (for example, 1 case per 10,000 population with a minum of resource use) can be researched.

An essential aspect of control strategies is the organization of leprosy control programmes, and their integration into the general health services. The different integration strategies, which are country-specific, can be compared in terms of effectiveness and costs. 


\section{Explanation of observed trends, and identification of research priorities}

Careful analysis of observed trends and results of existing control programmes can be used for monitoring the performance of a programme. This may also contribute to the improvement of our knowledge of the natural history and the transmission of leprosy. In this way, the approach may be used to identify research priorities. A better understanding of leprosy will in turn lead to better possibilities for rational decision making.

\section{SCIENTIFIC REQUIREMENTS}

It must be emphasized that a decision analytic approach to control questions does need validated models. Thus, two stages can be distinguished: (1) model development, and (2) model application (see Figure 1).

The development of validated models (stage 1) requires a collaborative effort with leprosy control projects which have longitudinal data. The use of such databases is indispensable. An ideal database comprises complete longitudinal data on diseased and also on healthy persons. It covers a complete and sufficiently large region, for which additional information, e.g. demographic, is available.

The data from such projects should first be used to estimate the parameters of the model, and then to test the joint action of all the factors. For example, models that are to be used for making predictions about trends (stage 2) should be validated by predicting historical trends as observed in the control projects. In practice, the initially validated model will be upgraded whenever new knowledge or relevant data become available.

\section{MODEL DEVELOPMENT}

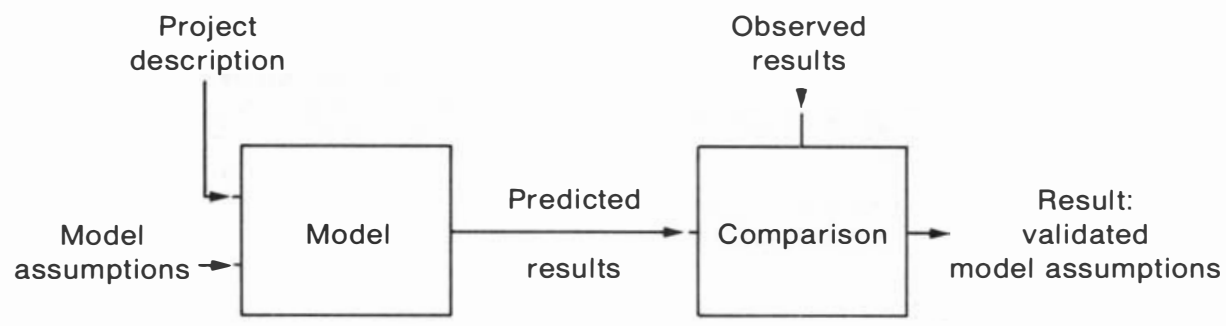

2 MODEL APPLICATION

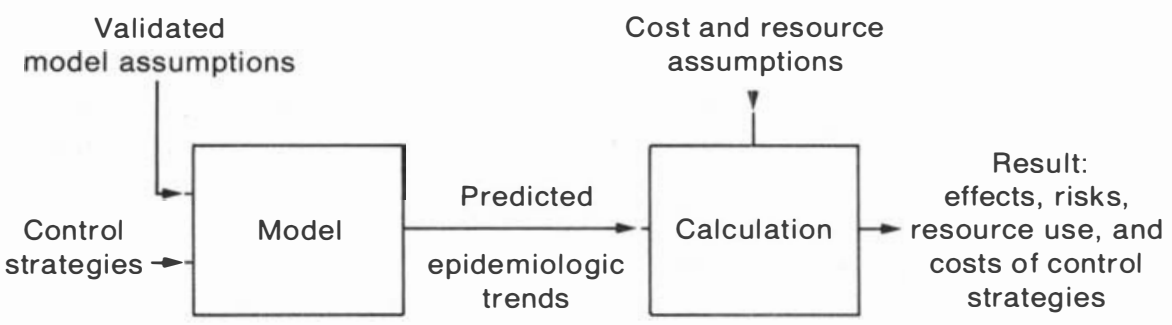

Figure 1. Model development (1) and application (2). 


\section{Conclusions}

The success of decision models as an aid in leprosy control cannot be guaranteed beforehand. But applied modelling could be important when addressing questions in prediction, planning, monitoring and evaluation. There are excellent control programmes with longitudinal data which could form the backbone for constructing validated simulation models. Those in charge of leprosy control programmes should seriously consider a collaboration with scientists and decision makers for jointly developing practical decision aids. , $^{9,11}$

\section{References}

${ }^{1}$ Gilbody JS. Impact of multidrug therapy on the treatment and control of leprosy. Int J Le pr, 1991; 59: 45878.

2 Maas PJ van der, Koning HJ de, Ineveld BM van, Oortmarssen GJ van, Habbema JDF et al. The costeffectiveness of breast cancer screening. Int J Cancer, 1989; 43: 1055-60.

${ }^{3}$ Oortmarssen GJ van, Habbema JDF, Maas PJ van der, Koning HJ de, Collette HJA, et al. A model for breast cancer screening. Cancer, 1990; 66: 1601-12.

4 Remme JHF et al. Epidemiological modelling for tropical disease control. Lepr Rev 1992; 63, Supplement, 40-7.

5 Plaisier AP, Oortmarssen GJ van, Remme JHF, Alley ES, Habbema JDF. The risk and dynamics of onchocerciasis recrudescence after cessation of vector control. Bull. WHO, 1991; 69: 169-78.

${ }^{6}$ Habbema JDF, Plaisier AP, Oortmarssen GJ van, Remme J, Alley ES. Prospective evaluation of onchocerciasis control strategies. Acta Leidensia, 1990; 59: 387-98.

7 Plaisier AP, Oortmarssen GJ van, Habbema JDF, Remme J, Alley ES. ONCHOSIM, a simulation model for the transmission and control of onchocerciasis. Computer Methods and Programs in Biomedicine, 1990; 31: 43-56.

${ }^{8}$ Lechat MF, Misson JY, Vellut CM, Misson CB, Bouckaert A. Un modèle épidémiométrique de la lèpre. Bull WHO, 1974; 51: 361-73.

9 Lechat MF, Misson CB, Bouckaert A, Vellut C. An epidemiometric model of leprosy: a computer simulation of various control methods with increasing coverage. Int J Lepr, 1977; 45: 1-8.

10 Lechat MF, Misson CB, Vanderveken M, Vellut CM, Declercq EE. A computer simulation of the effect of multidrug therapy on the incidence of leprosy. Ann Soc belga Med trop, 1987; 67: 59-65.

11 Lechat MF, Declercq EE, Misson CB, Vellut CM. Selection of MDT strategies through epidemiometric modelling. Int J Lepr, 1990; 58: 296-301.

Figure 1. Model development (1) and application (2). 